\title{
Capacidad reproductiva de Nannochloropsis oculata en diferentes concentraciones de salinidad y fertilizante: Una contribución a la Bioeconomía acuícola
}

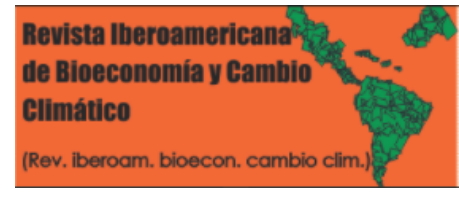

\section{Reproductive capacity of Nannochloropsis oculata in different concentrations of salinity and fertilizer: a contribution to aquaculture Bioeconomía}

\author{
Osorio, K; Palacios, K; Lumbi, D; Hsieh, P; Zuniga-González, Carlos \\ A; Aguilar, A; Editor Académico Dra. Claudia Zenteno-Ruiz
}

\author{
iD K Osorio \\ katherinne.osorio@ev.unanleon.edu.ni \\ Universidad Nacional Autónoma de Nicaragua, León \\ (UNAN-León)., Nicaragua \\ (iD) $\mathrm{K}$ Palacios \\ karen.palacios@ev.unanleon.edu.ni \\ Universidad Nacional Autónoma de Nicaragua, León \\ (UNAN-León)., Nicaragua \\ iD $\mathrm{D}$ Lumbi \\ dalia.lumbi@ev.unanleon.edu.ni \\ Universidad Nacional Autónoma de Nicaragua, León \\ (UNAN-León)., Nicaragua \\ P Hsieh \\ p.y.hsieh@icdf.org.tw \\ Misión Técnica de Taiwán en Nicaragua, International \\ Cooperation and Development Fund (ICDF)., Taiwán \\ (iD Carlos A Zuniga-González \\ czuniga@ct.unanleon.edu.ni \\ Universidad National Autonoma de Nicaragua, Leon., \\ Nicaragua
}

iD A Aguilar

arielaguilar@ev.unanleon.edu.ni

Universidad Nacional Autónoma de Nicaragua, León

(UNAN-León)., Nicaragua

Editor Académico Dra. Claudia Zenteno-Ruiz

Universidad Juárez Autónoma de Tabasco-

México. División académica de ciencias biológicas

(DACBIOL), México

Revista Iberoamericana de Bioeconomía y Cambio Climático

Universidad Nacional Autónoma de Nicaragua, León, Nicaragua ISSN-e: 2410-7980

Periodicidad: Semestral

vol. 6 , núm. 12,2020

czuniga@ev.unanleon.edu.ni
Resumen: El estudio se centró en contribuir a la Bioeconomía Acuícola evaluando el ritmo reproductivo de Nannochloropsis oculata en dos baterías experimentales con concentraciones salinas de $33 \%$, $25 \%$, $20 \%$, $15 \%$, $10 \%$ y $5 \%$. A cada una se le aplicó dosis únicas de $0.32 \%$ y $1 \%$ de F/2 Guillard, respectivamente. Se realizaron dos experimentos en tiempos de 10 y 23 días a temperatura de $25^{\circ} \mathrm{C}$. El primer experimento con $0.32 \%$ de F/2 Guillard, $N$. oculata presentó mayor número de cel $/ \mathrm{ml}$ en salinidades de $33 \%$ y $25 \%$, a los 8 días de estudio, denotando la afinidad de $N$. oculata de reproducirse con mayor velocidad en ese rango de salinidad. La importancia del uso de F/2 Guillard fue observada usando $1 \%$ debido a que la microalga presenta similar número de cel/ $\mathrm{ml}(\mathrm{P} \leq 0.05)$ en concentraciones salinas de $33 \%$, $25 \%$, $20 \%$ y $15 \%$ y similar concentración a las microalgas cultivadas con $0.32 \%$ de F/2 Guillard en salinidades de 33 \% y $25 \%$ o. La capacidad de $N$. oculata fue testado en un segundo experimento donde los resultados muestran similar número de cel/ $\mathrm{ml}$ entre los tratamientos salinos, en ambas baterías, a los 23 días de cultivo. Los resultados muestran que $N$. oculata puede ser cultivada con bajas concentraciones de F/2 Guillard en rangos de salinidad entre $25 \%$ y $33 \%$, implica reducción de costos.

Palabras clave: Batería, Salinidad, Temperatura, Nannochloropsis, Nutriente, Bioeconomía Acuícola.

Abstract: The study was focused on contributing to the Aquaculture Bioeconomy by evaluating the reproductive rhythm of Nannochloropsis oculata in two experimental batteries with saline concentrations of $33 \%$, $25 \%$, $20 \%$, $15 \%$, $10 \%$ o and $5 \%$. Single doses of $0.32 \%$ and $1 \%$ of F / 2 Guillard were applied to each one, respectively. Two experiments were carried out at times of 10 and 23 days at a temperature of $25^{\circ} \mathrm{C}$. The first experiment with $0.32 \%$ of F / 2 Guillard, N. oculata presented a higher number of cells / $\mathrm{ml}$ in salinities of $33 \%$ and $25 \%$, at 8 days of study, denoting the affinity of $N$. oculata to reproduce with greater speed in that salinity range. The importance of using F / 2 Guillard was observed using $1 \%$ because the microalgae has a similar number of cells $/ \mathrm{ml}(\mathrm{P} \leq 0.05)$ in saline concentrations of $33 \%$, $25 \%$, $20 \%$ and $15 \%$ and similar concentration to 
Recepción: 07 Julio 2020

Aprobación: 04 Noviembre 2020

URL: http://portal.amelica.org/ameli,
jatsRepo/394/3941759003/index.htm

DOI: https://doi.org/10.5377/ribcc.v6i12.9977

Autor de correspondencia: arielaguilar@ev.unanleon.edu.ni microalgae cultured with $0.32 \%$ of F / 2 Guillard in salinities of $33 \%$ and $25 \%$. The capacity of $N$. oculata was tested in a second experiment where the results show a similar number of cells / $\mathrm{ml}$ between the saline treatments, in both batteries, at 23 days of culture. The results show that $N$. oculata can be cultivated with low concentrations of $\mathrm{F} / 2$ Guillard in salinity ranges between $25 \%$ and $33 \%$, which implies cost reduction.

Keywords: Battery, Salinity, Temperature, Nannochloropsis, Nutrient, Aquaculture Bioeconomy.

\section{INTRODUCCIÓN}

Uno de los ejes fundamentales de la bioeconomía acuícola en los laboratorios de reproducción de peces se enfoca en la producción de alimento vivo (Almendarez, 2015) para disminuir costos operativos ante la alta demanda de alevines en las granjas de producción de peces marinos o de agua dulce, contribuyendo a la mejora de la rentabilidad de la actividad acuícola (FAO, 2014). La contribución de las microalgas a la bioeconomía acuícola se relaciona con la potencialidad que poseen estos microorganismos para mejorar la sostenibilidad costo-beneficio en la producción de alevines de peces debido a su alto contenido nutricional (Fernández et al., 2018). Las microalgas aportan polisacáridos, lípidos, aminoácidos, proteínas y vitaminas que varían en cantidad según la especie, por lo que son implementadas como mezcla para incrementar la calidad nutricional de los microorganismos usados como alimento vivo para larvas de peces (Lubzens et al., 1995).

Nannochloropsis oculata es una microalga que pertenece a la clase Eustigmatophyceae, se caracteriza por ser unicelular, con formas subesféricas o cilíndricas y miden entre 2 y $4 \mathrm{~mm}$, contiene clorofila "a" y otros pigmentos como astaxantina, zeaxantina y canthaxantina y los cloroplastos presentan coloración de

amarillo a verde (Guiry, 2013). Asimismo, este género agrupa especies que contienen la mayor cantidad de ácidos grasos poliinsaturados (PUFAs), especialmente ácido eicosapentaenoico (EPA), ácido araquidónico (ARA), docosahexaenoico (DHA) de gran importancia en la nutrición, formación del sistema nervioso y desarrollo de larvas de organismos marinos (Sánchez-Torres et al., 2008). El alto contenido de ácidos grasos poliinsaturados y su tolerancia a los cambios ambientales hace que esta especie de microalga sea ampliamente utilizada para alimentar rotíferos (Fulks y Main 1991; Renaud et al., 1991; Lubzens et. al. 1995; Lubían et. al. 2000) o para consumo humano (Sukenik, 1999; Barclay y Apt, 2013). Por consiguiente, estudiar los parámetros fisicoquímicos y contenido de nutrientes que mejoren el comportamiento reproductivo y calidad nutricional de las microalgas, contribuye al fortalecimiento de la bioeconomía acuícola (Fernández et al., 2018). Algunos autores muestran que Nannochloropsis oculata cultivada en ambientes abiertos, con una temperatura entre $12-15^{\circ} \mathrm{C}$ y concentración salina de 28-30 \%o llega a alcanzar concentraciones promedio de hasta $115 \times 10^{6} \mathrm{cel} / \mathrm{ml}$, mientras que a temperatura mayor a $20{ }^{0} \mathrm{C}$ alcanza valores de concentración entre 46 y 60 millones de cel/ml (Persíco et al., 2011). En ambientes abiertos con temperatura de $31{ }^{0} \mathrm{C}$ y 25 $\%$ de salinidad se han obtenido concentraciones de $75 \times 10^{6} \mathrm{cel} / \mathrm{ml}$, sugiriendo que la densidad celular de Nannochloropsis oculata cultivadas en ambientes abiertos presenta un ritmo de crecimiento inversamente proporcional a la temperatura (Álvarez-Lajonchére et al., 1996).

Por otro lado, en condiciones de laboratorio, es importante controlar la longitud de onda emitida por la luz artificial debido a que Nannochloropsis oculata contiene clorofila "a" y esta tiene rangos muy cortos de

\section{NotAS DE AUTOR}


absorción en los dos picos de máxima eficiencia fotosintética (Sánchez-Torres et al., 2008). En salinidades de $33 \%$ y usando lámparas fluorescentes de 40 wats, fotoperiodo 12:12 (luz - oscuridad), se han obtenido

concentraciones de $6.52 \times 10^{7} \mathrm{cel} / \mathrm{ml}$ en un periodo de 7 días (Paes et al., 2017). Ante tal situación, en diversos estudios se ha demostrado que Nannochloropsis oculata puede variar su comportamiento reproductivo o su calidad nutricional solamente por la variación de unos de los parámetros fisicoquímicos, tales como: contenido nutricional ofrecido en el medio de cultivo, longitud de onda o la concentración salina del medio de cultivo. Es más, las principales estrategias evolutivas sobre la capacidad reproductiva en momentos de escases de nutrientes que han adoptado las células vegetales se fundamentan en la capacidad de almacenar grandes cantidades del enzima ribulosa-1,5-bifosfato (RuBP) carboxilasa/oxigenasa (rubisco) (Andrew y Lorimer, 1987) y que los bioelementos invertidos en su síntesis pueden ser recuperados mediante catálisis en momentos de senescencia natural o inducida por estrés, o en una situación de carencia de nutrientes (Kozaki y Takeba, 1996 ; Ferreira et al., 2000). Por lo tanto, el objetivo de este trabajo fue evaluar la capacidad reproductiva de Nannochloropsis oculata usando dos baterías experimentales y aplicando dosis únicas de 0.32 \% y $1 \%$ de F/2 Guillard, respectivamente; en salinidades de 33\%o, 25 \%o, 20 \%o, 15 \%o, 10 $\%$ y $5 \%$, periodos de 10 y 23 días y temperatura de $25^{\circ} \mathrm{C}$.

\section{Materiales Y MÉTODOS}

\section{Microorganismo de estudio}

Se utilizó una cepa de Nannochloropsis oculata del cepario del Laboratorio de Investigaciones Marinas y Acuícolas (LIMA), Facultad de Ciencia y Tecnología de la Universidad Nacional Autónoma de Nicaragua, León (UNAN-León), ubicado en las Peñitas, León. En el desarrollo del trabajo de investigación se realizaron dos experimentos, cada uno estuvo estructurado por dos baterías experimentales con medios de cultivo de $250 \mathrm{ml}$ y 33 \%o, $25 \%$ o, $20 \%$ o, $15 \%$ o, 10 \% y 5 \%o de salinidad. Las baterías experimentales contenían

concentraciones de $0.32 \%$ y $1 \%$ de F/2 Guillard, respectivamente. Ambos experimentos se realizaron con temperatura de $25{ }^{\circ} \mathrm{C}$ y luminosidad constante de aproximadamente $250 \mu \mathrm{mol} \mathrm{E} / \mathrm{m}^{2} / \mathrm{s}$.

\section{Composición del fertilizante F/2 Guillard}

Tiene una composición final por litro: $75 \mathrm{mg} \mathrm{KNO}_{3}, 5.65 \mathrm{mg} \mathrm{NaH}{ }_{2} \mathrm{PO}_{4} \cdot 2 \mathrm{H}_{2} \mathrm{O}, 4.360 \mathrm{mg}$ EDTA.Na, $3.150 \mathrm{mg} \mathrm{FeCl}_{3} .6 \mathrm{H}_{2} \mathrm{O}, 0,010 \mathrm{mg} \mathrm{CuSO} 4.5 \mathrm{H}_{2} \mathrm{O}, 0,022 \mathrm{mg} \mathrm{ZnSO} 4.7 \mathrm{H}_{2} \mathrm{O}, 0,010 \mathrm{mg} \mathrm{CoCl} 2.6 \mathrm{H}_{2} \mathrm{O}, 0.180$ $\mathrm{mg} \mathrm{MnCl} \cdot 4 \mathrm{H}_{2} \mathrm{O}, 0,006 \mathrm{mg} \mathrm{Na} 2 \mathrm{MoO}_{4} \cdot 2 \mathrm{H}_{2} \mathrm{O}, 2 \mu \mathrm{g}$ cianocobalamina cristalina (B12), $0.100 \mathrm{mg}$ tiamina clorhídrica (B1), 0,001 mg biotina cristalina.

\section{Diseño experimental}

\section{Experimento 1, Tratamiento salino dependiente de la concentración}

Se formaron 2 baterías experimentales con dos concentraciones de F/2 Guillard (batería 1: 1 \%o y batería 2: $0.32 \%$ ), con seis concentraciones salinas ( $33 \%$, 25\%o, 20\%o, 15\%o, 10\%, y $5 \%$ ) cada batería y volúmenes de $250 \mathrm{ml}$, para evaluar la capacidad reproductiva de Nannochloropsis oculata. Previo al ensayo experimental, el agua de mar se pasó por un filtro UV y otro de papel de paso lento, para finalmente ser esterilizada. A partir del agua marina se realizaron las diluciones usando agua destilada. El tiempo de duración del experimento 
fue de 10 días. Temperatura e irradiancia fue como se describe anteriormente. El valor de salinidad se midió diario y se corregía con agua destilada.

\section{Experimento 2. Tratamiento tiempo dependiente de crecimiento}

Se formaron 2 baterías experimentales compuestas por 3 concentraciones salinas ( $33 \%$, 25\%o, 20\%o,), cada una, para evaluar el ritmo de crecimiento de Nannochloropsis oculata en concentraciones de F/2 Guillard

(batería 1: $1 \%$ y batería 2: $0.32 \%$ ). Tiempo de duración del experimento fue de 23 días. El procedimiento experimental fue similar al descrito en el experimento 1.

\section{Conteo celular}

Las densidades celulares se determinaron cada 24 horas mediante conteo en hemocitómetro o cámara de Neubauer de $0.1 \mathrm{~mm}$ de profundidad, expresándolas como número de células por mililitro (cel/ml). Las lecturas se realizaron por triplicado, se sumó el número de células contadas en los cuatro cuadrantes externos de la cámara y se dividió entre 2500. Este procedimiento se usó para contar el número de cel $/ \mathrm{ml}$ en los tratamientos salinos definidos en las dos baterías.

\section{Análisis estadisticos}

Las comparaciones entre las concentraciones salinas en cada batería experimental se realizaron mediante ANOVA de una vía y entre baterías experimentales se usó ANOVA de dos vías (SigmaStat; SPSS Inc., Chicago, IL). Previo al análisis de varianza, los datos fueron analizados mediante una prueba de normalidad (Shapiro-Wilks) y de homogeneidad de varianzas (prueba C de Cochran). Las comparaciones post hoc se realizaron mediante una prueba de Student-Newman-Keuls. Los datos se muestran como media \pm E.E.M. de cada grupo y las diferencias se consideraron estadísticamente significativas a $\mathrm{P}<0,05$.

\section{RESULTADOS Y DISCUSIÓN}

\section{Primer experimento:}

De manera general, nuestros resultados muestran que donde se aplicó $0.32 \%$ de F/2 Guillard los medios con concentraciones salinas de $33 \%$ y $25 \%$ presentaron la mayor cantidad de cel $/ \mathrm{ml}$ de Nannochloropsis oculata, a los 8 días de estudio (figura 1), denotando la afinidad de Nannochloropsis oculata 


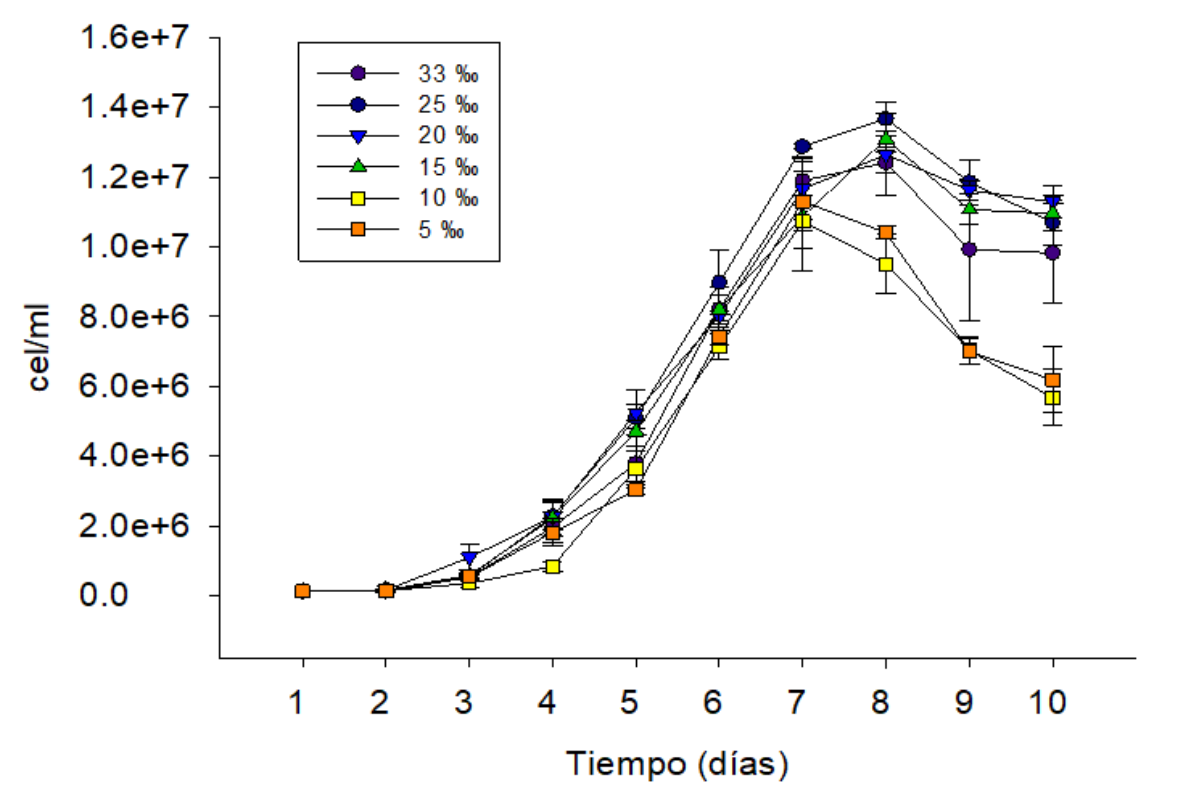

FIGURA 1

Ritmo de reproducción de Nannochloropsis oculta, cultivada con $0.32 \%$ F/2 Guillard y concentraciones salinas( $33 \%$, $25 \%$, $20 \%$, $15 \%$, $10 \%$, $5 \%$ ),en un período de 10 días.

Este resultado coincide con lo reportado en estudios recientes, donde usando fertilizante agrícola y vitaminas del complejo B obtuvieron resultados similares a los nuestros, la fase de latencia se mantiene entre

3-4 días y la fase exponencial se prolonga hasta los ocho días (Pereira et al 2017). Otros estudios muestran que Nannochloropsis oculata presenta comportamiento reproductivo similar al encontrado en nuestro estudio cuando se encuentra cultivada en medio con salinidad de 28-30 \%o (Persico et al., 2011). Bajo este contexto, nuestros resultados invitan a sugerir que Nannochloropsis oculata puede cultivarse con $0.32 \%$ $\mathrm{de} \mathrm{F} / 2$ Guillard,en un volumen de $250 \mathrm{ml}$ y obtener razonable rendimiento reproductivo, en concentraciones de $33 \%$ y $25 \%$ de salinidad y temperatura de $25{ }^{\circ} \mathrm{C}$. Por otro lado, los efectos generalizados sobre el comportamiento reproductivo de Nannochloropsis oculata en la batería experimental donde se agregó 1 \%o de F/2 Guillard (figura 2), confirman la importancia de la aplicación de este fertilizante para la reproducción de esta microalga (Martínez et al., 2017) cuando es cultivada en concentraciones salinas menores a $25 \%$ o (Sánchez-Torres et al., 2008). 


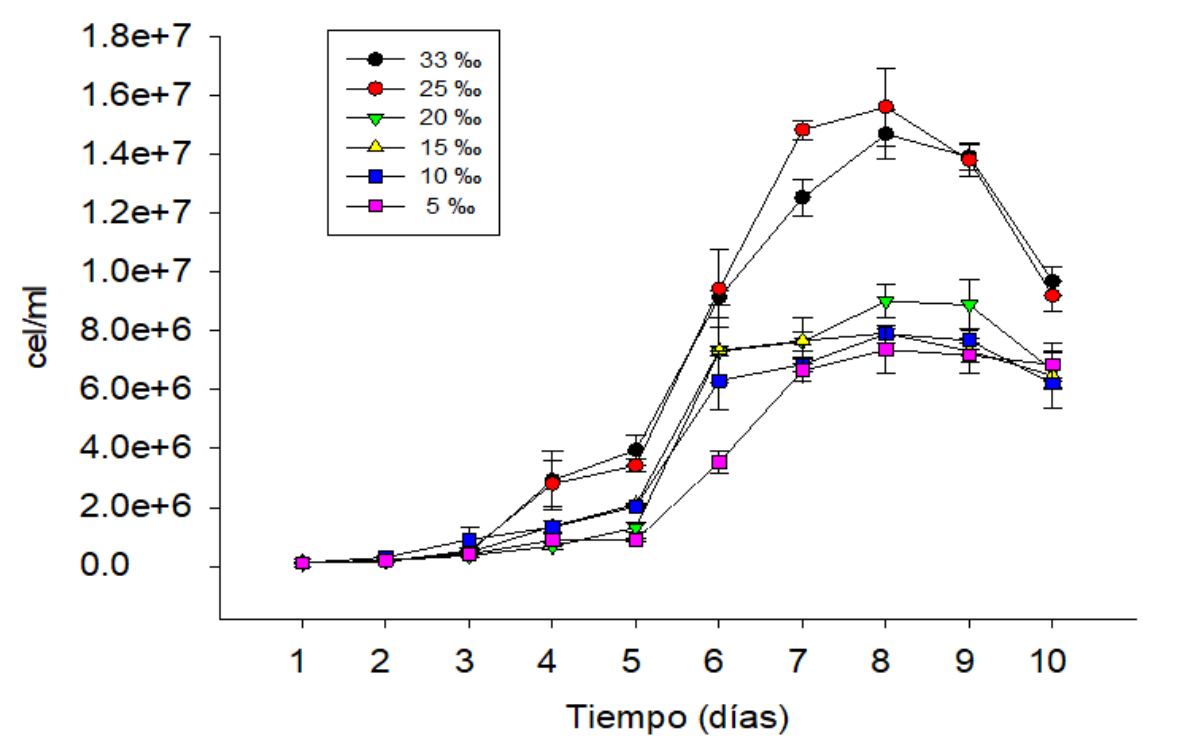

FIGURA 2.

Ritmo de reproducción de Nannochloropsis oculta, cultivada con $1 \%$ F/2 Guillard y concentraciones salinas(33\%o, $25 \%$, $20 \%$, $15 \%$, $10 \%$, $5 \%$ )en un período de 10 días.

Por lo tanto, nuestros datos muestran que Nannochloropsis oculata puede ser cultivada en un amplio rango de salinidades, coincidiendo con lo reportado por otros investigadores (Álvarez- Lajonchére et. al., 1996; Ra et. al., 2016). Además, hemos demostrado que el ritmo reproductivo de Nannochloropsis oculata es similar cuando se encuentra cultivada en medio con $33 \%$ y $25 \%$ y usa $0.32 \%$ o $1 \%$ o de F/2 Guillard (figura 3), denotando la afinidad a la concentración salina oceánica (Pereira et al., 2017) y a la capacidad de reproducción con escaza presencia de nitrógeno (Paez et al., 2016), quizás debido a mecanismos contra reguladores como la catálisis de la enzima rubisco (ribulosa-1,5-bisfosfato carboxilasa/oxigenasa) para abastecer a la célula de nitrógeno, azufre y carbono en momentos de carencia de nutrientes (Andrews y Lorimer, 1987; Ferreira et al., 2000). Por consiguiente, obtener igual densidad poblacional en los medios de cultivo con $0.32 \%$ y $1 \%$ de F/2 Guillard en las concentraciones de $33 \%$ y $25 \%$ muestran la capacidad de reproducción de Nannochloropsis oculata bajo limitaciones de nitrógeno. Sin embargo, si se incrementa la concentración de fertilizante de $0.32 \%$ a $1 \%$, la reproducción de Nannochloropsis oculata no se ve afectada por la salinidad en un rango de $15 \%$ a $33 \%$. Es más, los medios de cultivo con $5 \%$, $10 \%$, $15 \%$ y $20 \%$ o que contienen $1 \%$ o de $\mathrm{F} / 2$ Guillard presentan mayor concentración celular que los medios de cultivo con idénticas concentraciones salinas y $0.32 \%$ de F/2 Guillard (figura 3). 


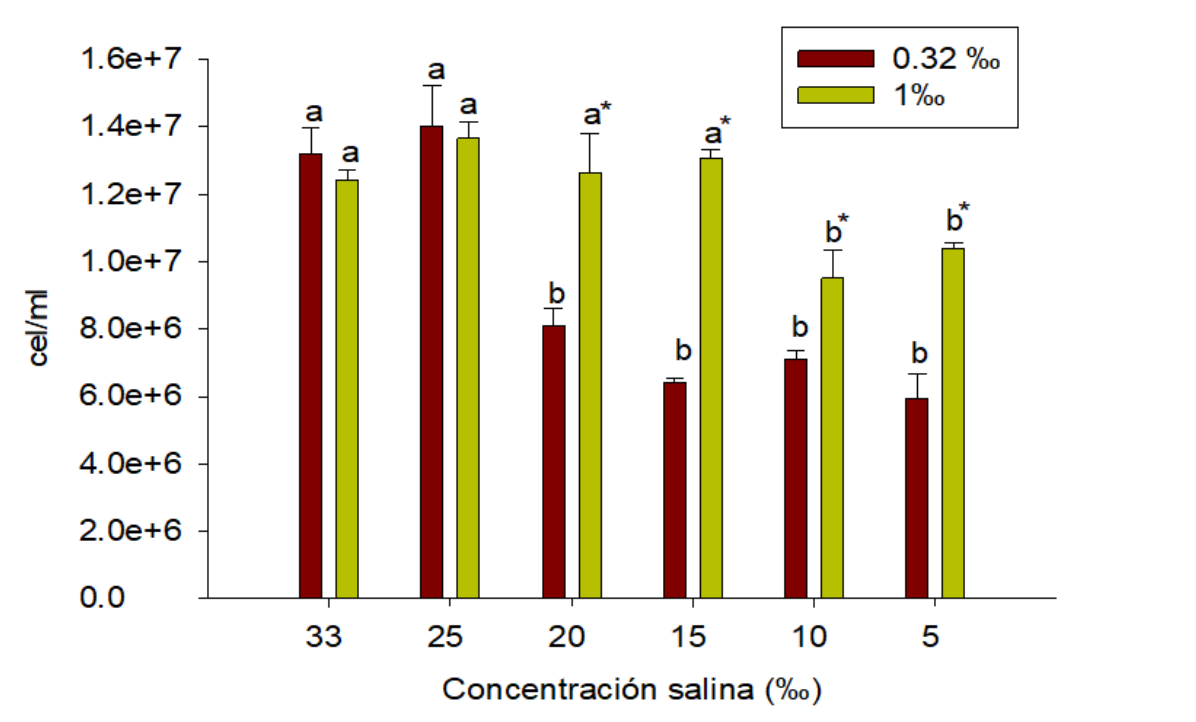

FIGURA 3.

Cantidad de células por ml de Nannochloropsis oculta, cultivada con $0.32 \%$ y $1 \%$ de F/2 Guillard enconcentraciones salinas (33\%, $25 \%$, $20 \%$, $15 \%$, $10 \%$, $5 \%$ ), 8 días de cultivo. Letras diferentes indican diferencias significativas, de manera individual, para cada batería experimental. *: Indica diferencias significativas entre los tratamientos con $0.32 \%$ y $1 \%$ de F/2 Guillard en una misma concentración salina.

Situación que pudiese estar siendo mediada por los cofactores vitamínicos presente en F/2 Guillard (Pereira et al., 2017). Además, se observa igual periodo de tiempo para la reproducción de la microalga en la fase de adaptación (0-4 días) y exponencial (5-9 días), lo cual coincide con lo reportado por otros investigadores (Martínez et. al., 2017; Pereira et. al., 2017; Ra et. al., 2016). Cabe mencionar, que en nuestro experimento se agregó una sola dosis inicial de F/2 Guillard, a diferencia de lo realizado por Martínez et al., (2017) que agregó el fertilizante cada 4 días. Por lo tanto, nuestros resultados muestran que una sola dosis de $0.32 \%$ de F/2 Guillard es suficiente para tener una concentración de microalgas de $13 \times 10^{6} \mathrm{cel} / \mathrm{ml}$, en un medio de cultivo con volumen de $250 \mathrm{ml}$ en un periodo de 8 días, temperatura de $25{ }^{0} \mathrm{C}$ y concentración salina de $25 \%$ o $33 \%$, teniendo concentración inicial de $12 \times 10^{4} \mathrm{cel} / \mathrm{ml}$ de Nannochloropsis oculata.

\section{Segundo experimento:}

Tras determinar que $\mathrm{N}$. oculata se reproduce bien en concentraciones salinas de $33 \%$, $25 \%$ y concentraciones de 0.32 \% y $1 \%$ de F/2 Guillard se evaluó su capacidad reproductiva en un periodo de 23 días, bajo las mismas condiciones experimentales descritas en el primer experimento en salinidades de $33 \%$, $25 \%$ y $20 \%$ o. Nuestros resultados muestran similar ritmo reproductivo entre los tratamientos salinos de cada batería experimental y los tratamientos salinos entre concentraciones de F2 Guillard de ambas baterías (Figura 4.) 
A

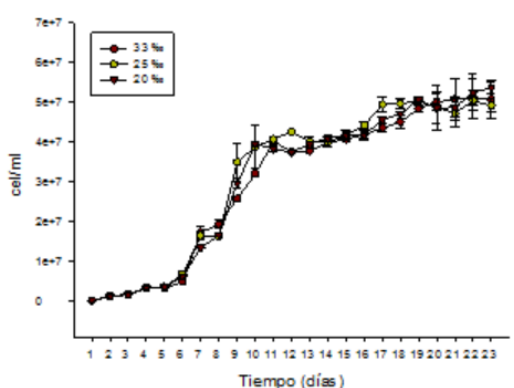

B

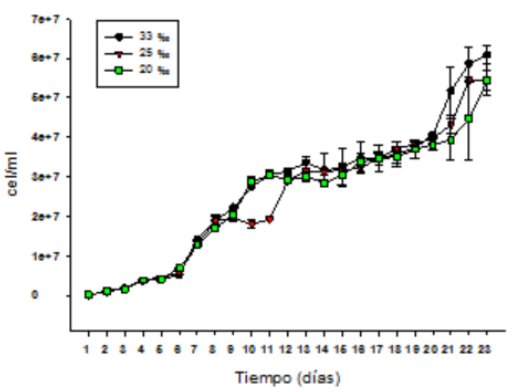

FIGURA 4

Ritmo de reproducción de Nannochloropsis oculta cultivada en diferentes contracciones salinas $(33 \%, 25 \%, 20 \%, 15 \%, 10 \%, 5 \%)$ Periodo de 23 dias A: 0.32 \%,F/2 Guillar, B>1 F/2 Guillard.

Algunos investigadores han cultivado $N$ oculata por 23 días, con niveles de irradiancia de 650 y $400 \mu \mathrm{mol}$ $\mathrm{E} / \mathrm{m}^{2} / \mathrm{s}$, temperatura de $25^{\circ} \mathrm{C}$ y aplicación $\mathrm{F} / 2$ Guillard cada 4 días y los resultados muestran que la densidad celular de los cultivos alcanzan valores de hasta $175 \pm 1.83$ y $148 \pm 1.97 \times 10^{6} \mathrm{cel} / \mathrm{ml}$, respectivamente. Mientras que en niveles de irradiancia de 80 y $200 \mu \mathrm{mol} \mathrm{E} / \mathrm{m}^{2} / \mathrm{s}$ la densidad celular alcanza valores de $21 \pm$ 1.12 y $43 \pm 1.84 \times 10^{6} \mathrm{cel} / \mathrm{ml}$, respectivamente (Martínez et al., 2017). Nuestro ensayo experimental se hizo bajo las mismas condiciones del estudio realizado por Martínez et al., (2017) con nivel de irradiancia de

aproximadamente $250 \mu \mathrm{mol} \mathrm{E} / \mathrm{m}^{2} / \mathrm{s}$ con la excepción que nosotros realizamos una sola aplicación de F/2 Guillard a cada batería experimental (0.32\%o y $1 \%$ ), al inicio del experimento. No obstante, resulta interesante observar que, aunque el F/2 Guillard solo se aplicó al inicio del experimento se logró obtener densidades de cel $/ \mathrm{ml}$ similares en las tres concentraciones salinas, en cada batería y entre baterías (Figura 5).

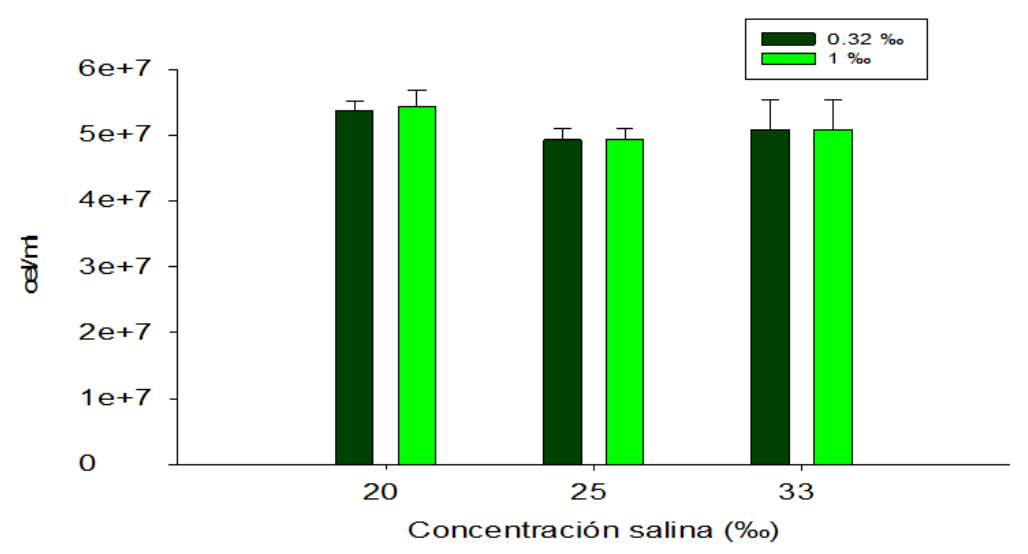

FIGURA 5

Cantidad de celulas por ml de Nannocholoropsis oculta, cultivada con $0.32 \%$ y 1

$\%$ de F/2 Guillard en concentraciones salinas (33 \%, y $20 \%$ ), 23 días de cultivo.

Por lo tanto, observar rendimientos similares de la concentración de cel/ml y del comportamiento reproductivo, entre ambas baterías experimentales, a lo largo de 23 días de cultivo sugiere que Nannochloropsis oculata puede ser cultivada con $0.32 \%$ de $\mathrm{F} / 2$ y que esta concentración de fertilizante es suficiente para obtener rendimientos de hasta $50 \times 10^{6} \mathrm{cel} / \mathrm{ml}$, independientemente del agotamiento del nitrógeno. Es 
más, que esta microalga puede continuar reproduciéndose posiblemente a la capacidad de protección celular frente al estrés que presenta la vía fotorrespiratoria (Xiao et. Al., 2013; Barclay y Apt, 2013) debido a la acumulación de grandes cantidades del enzima rubisco y que los bioelementos invertidos en su síntesis pueden ser recuperados por medio de las rutas catabólicas de rubisco que se activan cuando se produce una decadencia funcional

durante la senescencia natural o inducida por estrés, o en una situación de carencia de nutrientes (Ferreira et. al., 2000; Wei et. al., 2017). Por consiguiente, el aplicar una sola dosis del fertilizante F/2 Guillard al inicio del experimento y observar igual cantidad de cel $/ \mathrm{ml}$ a los 23 días, entre las diferentes concentraciones salinas de cada batería (33 \%o, 25 \% y $20 \%$ ) y entre las concentraciones de F/2 Guillard de las dos baterías experimentales $(0.32 \%$ y $1 \%$ o), sugiere que, independientemente, de la escases de nitrógeno Nannochloropsis oculata podría continuar reproduciéndose debido a la característica del enzima rubisco de servir como una importante reserva de nitrógeno, azufre y carbono; elementos esenciales que la célula puede redirigir hacia otros destinos en situaciones críticas, de manera similar a lo reportado por otros investigadores (Paez et al., 2016; Wei et. al., 2017).

Por lo antes descrito, es importante tomar en cuenta lo mencionado por Anderson y Seijo (2010) y Alzugaray, R., Puga, R., Valle, S., Morales, O., Grovas, A., López, L. y Fujita, R. (2019) donde argumentan la necesidad de abordajes multidisciplinario en el enfoque de la Bioeconomía y de ahí la importancia de generar otros estudios en la Bioeconomía Acuícola que permitan conocer cuáles son los beneficios en rentabilidad para la producción de $N$. oculata, ¿o cuál es el manejo actual a nivel piloto comercial en los laboratorios de producción de juveniles de peces marinos? ¿Cuánto se necesita producir de esta microalga? y ¿Cuánto me ahorro con la implementación de estos manejos?

\section{ConCLUSIón}

En conclusión y contribuyendo a la gestión del conocimiento de la Bioeconomía Acuícola, los resultados presentados en este estudio confirman que Nannochloropsis oculata presenta mejor ritmo de crecimiento poblacional en concentraciones salinas de $25 \%$ y $33 \%$ y que en estas concentraciones salinas la densidad poblacional, a los 8 días de cultivo, presenta similar número de cel $/ \mathrm{ml}$ si se cultiva con $0.32 \%$ o $1 \%$ de F/2 Guillard. Asimismo, se confirma la importancia de este fertilizante cuando la microalga es cultivada en concentraciones salinas menores a $25 \%$. El trabajo demuestra que Nannochloropsis oculata puede reproducirse y alcanzar densidad poblacional de más de $50 \times 10^{6} \mathrm{cel} / \mathrm{ml}$, en un periodo de 23 días, con una sola dosis inicial de fertilizante; ya sea con $0.32 \%$ o $1 \%$ de F/2 Guillard y concentraciones salinas de 20 $\%$, $25 \%$ y $33 \%$, denotando la capacidad de $N$. oculata de reproducirse en medios con escaso contenido de nutriente.

Por consiguiente, cuando $N$. oculata se encuentra cultivada en ese rango de concentración salina y $0.32 \%$ y $1 \%$ o de F/2 Guillard presenta igual densidad poblacional (ver tabla 1) 
TABLA 1.

Comportamiento reproductivo de $N$. oculata en diferentes concentraciones de salinidad y dos concentraciones de F/2 Guillard (0.32 \%o y $1 \%$ ), en 10 y 23 días de cultivo.

\begin{tabular}{llll}
\hline \multicolumn{4}{l}{ ler experimento (10 dias de cultivo) } \\
\hline Concentración & Dosis F/2 Guillard (\%) & \\
salina (\%) & 0.32 & 1 & 0.32 vrs 1 \\
\hline 33 & $\mathrm{a}^{*}$ & $\mathrm{a}^{*}$ & $=$ \\
25 & $\mathrm{a}^{*}$ & $\mathrm{a}^{*}$ & $=$ \\
20 & $\mathrm{~b}$ & $\mathrm{a}^{*}$ & $\neq+$ \\
15 & $\mathrm{~b}$ & $\mathrm{a}^{*}$ & $\neq+$ \\
10 & $\mathrm{~b}$ & $\mathrm{~b}$ & $\neq+$ \\
5 & $\mathrm{~b}$ & $\mathrm{~b}$ & $\neq+$ \\
\hline 2 do experimento (23 dias de cultivo) & \\
\hline 33 & $\mathrm{a}$ & $\mathrm{a}$ & $=$ \\
25 & $\mathrm{a}$ & $\mathrm{a}$ & $=$ \\
20 & $\mathrm{a}$ & $\mathrm{a}$ & $=$ \\
\hline
\end{tabular}

\footnotetext{
Letras diferentes indican diferencias significativas entre tratamientos (salinidades) de cada una de las baterías experimentales ( $0.32 \%$ y $1 \%$ de F/2 Guillard). *: Indica mayor concentración de cel $/ \mathrm{ml}$, solo entre tratamiento de la misma batería. =: Indica similar concentración de cel/ml entre los tratamientos con concentraciones de 0.32 \% y 1 \% de $\mathrm{F} / 2$ Guillard, en una misma salinidad. $\neq+$ : Indica que la concentración de cel/ml en el medio con $1 \% 0>0.32 \%$ de F/2 Guillard en la misma salinidad.
}

Finalmente, el presente estudio contribuye a la Bioeconomía Acuícola debido a que la capacidad de reproducción de Nannochloropsis oculata con el consumo de poco nutrientes favorece la disminución de los costos de producción, es decir el cultivar la especie bajo estas condiciones conllevaría a una Bioeconomía más rentable, Spolaore, P., Joanni s - Cassan, C., Duran, E., y Isambert, A. (2006).

\section{Agradecimientos}

El equipo de investigación del Proyecto Pargo de la Universidad Nacional Autónoma de Nicaragua, León (UNAN-León) agradecemos el apoyo brindado, para el desarrollo de esta investigación, a las instituciones del gobierno de la Republica de Nicaragua: Instituto Nicaragüense de la Pesca y Acuicultura (INPESCA); Ministerio del Ambiente y los Recursos Naturales (MARENA) por ser parte activa de nuestro proyecto; a las empresas Torrecilla SEAJOY y Semillas Acuáticas, S,A por apoyarnos en la capacitación de personal y

donación de equipos; a la Unión Europea (UE) y Agencia Española de Cooperación Internacional para el Desarrollo (AECID) debido a que con los proyectos "fortalecimiento al desarrollo económico de cooperativas y mipymes que participan en 7 cadenas de valor incluyentes, aplicando enfoques de sostenibilidad y adaptación al cambio climático y de igualdad de género de la producción agropecuaria y pesquera en las zonas vulnerables ante la sequia de las segovias y occidente del pais" y "Fortalecimiento de capacidades para la cria y engorde en jaulas flotantes de pargo (Lutjanus sp) en 8 comunidades costeras de Nicaragua", respectivamente, se logró reconstruir una parte del Laboratorio de Investigaciones Marinas y Acuícola (LIMA) de la UNANLeón y adquirir algunos equipos; al Consejo Nicaragüense de Ciencia y Tecnología (CONICYT) por su aporte a la adquisición de equipos y reactivos; a la Cooperación Suiza para el Desarrollo (COSUDE) y Comisión Centroamericana de Ambiente y Desarrollo - SICA (CCAD-SICA) por su apoyo para la obtención de reactivos de investigación; a la Misión Técnica Taiwán en Nicaragua- ICDF- por colaborar con el grupo de investigación del proyecto pargo en los trabajos de investigación y apoyar en el mantenimiento y funcionamiento del LIMA. A Carmen Rojo y Pilar Ruiz (Universidad de Valencia, España) y Emilia Calvo y Jorge Boza (Universidad Nacional, UNA-Puntarenas- Costa Rica) por su valioso aporte a la capacitación del equipo de investigación del Proyecto Pargo. 


\section{REFERENCIAS CITADAS}

Alzugaray, R., Puga, R., Valle, S., Morales, O., Grovas, A., López, L., y Fujita, R. (2019). Un enfoque multiinstitutional para modelar el beneficio bioeconómico de perspectivas de manejo pesquero en Cuba. Revista Cubana de Investigaciones Pesqueras, 36(2), 0138-8452.

Almendarez, L. C. (2015). La Bioeconomía acuícola como herramienta para la toma de decisiones empresariales. ContactoS, 98, 14-18.

Anderson, L. y Seijo, J. 2010. Bioeconomics of Fisheries Management. Wiley-Blackwell, NJ. 305p.

Álvarez-Lajonchére, L., Hernández, O., Comas, A., Martínez, V., y Lozano, B. (1996). Efecto de la reducción de salinidad sobre la tolerancia a altas temperaturas en la microalga Nannochloropsis oculata. Hidrobiológica, 6(1-2), $39-42$.

Andrews, T., y Lorimer, G. (1987). Rubisco: Structure, mechanisms and prospects for improvement. En The Biochemistry of Plants, Vol. 10 (Hatch, M. D. and Boardman, N. K., Eds.) pp 131-218, Academic Press, San Diego. https://doi.org/10.1016/B978-0-12-675410-0.50009-9

Barclay, W. y Apt, K. (2013). The microalgae cell with reference to mass cultures: Strategies for bioprospecting microalgae for potential commercial applications. En: Richmond A, Hu Q, editores. Handbook of microalgal culture: applied phycology and biotechnology. 2a ed. Wiley Blackwell, (2013) 69-79. https:// doi.org/10.1002/9781118567166.ch4

Fao, W. F. P. (2014). IFAD (2012) The State of Food Insecurity in the World 2012: Economic growth is necessary but not sufficient to accelerate reduction of hunger and malnutrition. FAO, Rome.

Fernández, F., Sevilla, J. y Grima, E. (2018). Contribución de las microalgas al desarrollo de la bioeconomía. Mediterráneo económico, (31), 309-331.

Ferreira, R., Esquivel, M. y Teixeira, A. (2000). Catabolism of ribulose bisphosphate carboxylase from higher plants. Current Topics in Phytochemistry, 3, 129-165.

Fulks, W. y Main, K. (1991). Rotifer and microalgae culture systems. Proceeding of a US-Asia Workshop. Honolulu, Hawaii: The Oceanic Institute, p.1-364.

Guiry, M. (2013). AlgaeBase. World-wide electronic publication, National University of Ireland, Galway. http:// www.algaebase.org/search/genus/detail/?genus_id=44568; Recuperado en 04 Noviembre 2013

Kozaki, A., y Takeba, G. (1996). Photorespiration protects C3 plants from photooxidation. Nature 384, 557-560. https://doi.org/10.1038/384557a0

Lubian, L., Montero, O., Moreno-Garrido, I., Huerstas, I., Sobrino, C., González, M., y Pares, G. (2000). Nannochloropsis (Eustigmatophyceae) as source of commercially valuable pigments. J. Appl. Phycol. 12, 249-255. https://doi.org/10.1023/A:1008170915932

Lubzens, E., Gibson, O, Mora, O., y Sukenic, A. (1995). Potential advantages of frozen algae (Nannochloropsis sp.) for rotifer (Brachionus plicatilis) culture. Aquaculture 133, 295-310. https:// doi.org/10.1016/0044-8486(95)00010-Y

Martínez, M., Sanchez, R., Meza, E., Ulloa, R., y Saldivar, J. (2016). Síntesis de lípidos de la microalga Nannochloropsis oculata para su uso potencial en la producción de biodiesel. Rev. Int. Contam. Ambie. 33(85-91). https:// doi.org/10.20937/RICA.2017.33.esp02.08

Paes, C., Faria, G., Tinoco, N., Castro, D., Barbarino, E., y Laurenco, S. (2016). Growth, nutrient uptake and chemical composition of Chlorella sp. and Nannochloropsis oculata under nitrogen starvation. Lat. Am. J. Aquat. Res, 44(2), 275-292. DOI: 10.3856/vol44-issue2-fulltext-9. https://doi.org/10.3856/vol44-issue2-fulltext-9

Pereira, M., Jáuregui, G., Devia, A., y Rojas, J. (2017). Cultivo de microalgas Isochrysis galbana y Nannochloropsis sp. para alimentación de larvas de peces marinos. Mutis, 7(2), 81-85, doi: http:// dx.doi.org/10.21789/22561498.1246

Pérsico, M., Moris, M., Tranier, E., Zanazzi, A., Saubidet, A., y Beligni, M. (2011). Evaluación de un sistema exterior de cultivo masivo de la microalga marina Nannochloropsis oculata, en una zona templada oceánica de Argentina. Rev Lattiinoam Biiottecnoll Amb Allgall. 2(1), 30-48. 
Ra, C., Kang, C., Jung, J., Jeong, G., y Kim, S. (2016). Effects of light-emitting diodes (LEDs) on the accumulation of lipid content using a two-phase culture process with three microalgae. Bioresource Technology, 212, 254-261. https://doi.org/10.1016/j.biortech.2016.04.059

Renaud, S., Parry, D., Thinh, L. Kuo, C., Padovan, A., y Sammy, N. (1991). Effect of light intensity on the proximate biochemical and fatty acid composition of Isochrysis sp. and Nannochloropsis oculata for use in tropical aquaculture. J. Appl. Phycol. 3 (1), 43-53. DOI: 10.1007/BF00003918. https://doi.org/10.1007/BF00003918

Sánchez-Torres, H., Juscamaita-Morales, J., Vargas-Cárdenas, J., y Oliveros-Ramos, R. (2008). Producción de la microalga Nannochloropsis oculata (Droop) Hibberd en medios enriquecidos con ensilado biológico de pescado. Ecología Aplicada, 7(1-2), 149-158. https://doi.org/10.21704/rea.v7i1-2.370

Sukenik, A. (1999). In: COHEN Z, (Ed). Chemicals from Microalgae. London: Taylor y Francis, pp. 41-56.

Wei, L., Wang, Q., Xin, Y., Lu, Y., y Xu, J. (2017). Enhancing photosynthetic biomass productivity of industrial oleaginous microalgae by overexpression of rubisco activase. Algal Research. http://dx.doi.org/10.1016/ j.algal.2017.07.023

Spolaore, P., Joannis - Cassan, C., Duran, E., \& Isambert, A. (2006). Optimization of Nannochloropsis oculata growth using the response surface method. Journal of Chemical Technology \& Biotechnology: International Research in Process, Environmental \& Clean Technology, 81(6), 1049-1056. https://doi.org/10.1002/ jctb.1529

Xiao, Y., Zhang, J., Cui, J., Feng, Y., y Cui, Q. (2013). Metabolic profiles of Nannochloropsis oceanica IMET1 under nitrogen-deficiency stress. Bioresource Technology 130, $731-738$ https://doi.org/10.1016/ j.biortech.2012.11.116 